\title{
The Stability Analysis of Two-Species Competition Model with Stage Structure and Diffusion Terms
}

\author{
Wang Hailing \\ Institute of Information Science and Technology, Xiamen University Tan Kah Kee Colledge, Xiamen, China
}

Email address:

whling@xujc.com,19099428@qq.com

To cite this article:

Wang Hailing. The Stability Analysis of Two-Species Competition Model with Stage Structure and Diffusion Terms. Applied and Computational Mathematics. Vol. 5, No. 5, 2016, pp. 193-201. doi: 10.11648/j.acm.20160505.12

Received: September 6, 2016; Accepted: October 7, 2016; Published: October 13, 2016

\begin{abstract}
In this paper, the author proposed and considered a reaction-diffusion equation with diffusion terms and stage structure. We discussed the stability of the positive equilibrium. By using the upper-lower solutions and monotone iteration technique, we obtained the zero steady state and the boundary equilibrium were linear unstable and the unique positive steady state was globally asymptotic stability. The traditional results are improved and this result applies to broader frameworks.
\end{abstract}

Keywords: Stage Structure, Reaction-Diffusion Equations, Equilibrium, Stability

\section{Introduction}

The growth of the species, often needs a process of development. Meanwhile, different species with different growth stage showed the different characteristics. So, studying the population model with stage structure has practical significance. But, a single population is not too much, each species must be affected by the other populations and the environment, so, in recent years, the literature on the two phase structure of single population was more, see [1, 2,3, 4, 5] etc. Among them, Chen Lansun in literature [1] listed some scholars' research results. But, the stability of the equilibrium point on two population model was studied through ordinary differential equations in $[2,3,4,5]$. Later, many scholars began to research the structure of three phase single population model. Gao Shujing [6] set up for a class of population model according to the young adults aged three stages. In 2005, Liang etc [7] established a class of population model which was divided into a pupa Larvae, adult three phase structure, $\mathrm{Wu}$ [8] studied the global asymptotic stability of a weakly-coupled reaction diffusion system in the three-species model. But in these papers, the authors did not notice the time delay. In fact, the development of population reproduction has some lag process, literature [9, 10, 11] considered the time delay on the basis of $[6,7]$, but, in these papers the author did not notice the free diffusion phenomena of the population. Literature [12] studied two species predator-prey with stage structure and diffusion terms which considered the effect of diffusion and phase structure. But the authors considered the species only spreading in the local scope. The local operator did not accurately describe the object of study behavior of space and time, therefore, we must introduce the convolution operator to describe the spatial diffusion process. On the basis of [12], we considered the following competition model with diffusion terms and stage structure:

$$
\left\{\begin{array}{l}
\frac{\partial u_{1}(x, t)}{\partial t}-d_{1} \frac{\partial^{2} u_{1}(x, t)}{\partial x^{2}}=-a_{1} u_{1}(x, t)+\alpha u_{2}(x, t)-\alpha\left(\left(g_{1} * u_{2}\right)(x, t)\right) \\
\frac{\partial u_{2}(x, t)}{\partial t}-d_{2} \frac{\partial^{2} u_{2}(x, t)}{\partial x^{2}}=\alpha\left(\left(g_{1} * u_{2}\right)(x, t)\right)-b_{1} u_{2}(x, t)-r_{1} u_{2}^{2}(x, t)-c_{1} u_{2}(x, t) u_{3}(x, t) \\
\frac{\partial u_{3}(x, t)}{\partial t}-d_{3} \frac{\partial^{2} u_{3}(x, t)}{\partial x^{2}}=-b_{2} u_{3}(x, t)-r_{2} u_{3}^{2}(x, t)-c_{2} u_{2}(x, t) u_{3}(x, t)+\beta\left(g_{2} * u_{3}\right)(x, t)
\end{array}\right.
$$


Where, $u_{1}(x, t), u_{2}(x, t)$ respectively represent the population densities of the juvenile and adult of $\mathrm{A}$ at time $\mathrm{t}$ and location $\mathrm{x}$, $u_{3}(x, t)$ represent the population densities of $\mathrm{B}$ at time $\mathrm{t}$ and location $\mathrm{x}, d_{1}, d_{2}, d_{3}$ represent the diffusion rates, $a_{1}, b_{1}, b_{2}$ represent the death rate, $\alpha, \beta$ represent the birth rate, $r_{1}, r_{2}$ represent the density coefficient, $c_{1}, c_{2}$ represent the competition coefficient of adult A, B, The kernel $g_{1}(s), g_{2}(s)$ are inter-tribal and non-negative function satisfying

$$
\begin{gathered}
\int_{0}^{+\infty} g_{1}(s) d s=\int_{0}^{+\infty} g_{2}(s) d s=1,\left(g_{1} * u_{2}\right)(x, t)=\int_{0}^{+\infty} \int_{-\infty}^{+\infty} g_{1}(s) e^{-\gamma_{1} s} \frac{1}{\sqrt{4 \pi d_{2} s}} e^{-\frac{(x-y)^{2}}{4 d_{2} s}} u_{2}(t-s, y) d y d s \\
\left(g_{2} * u_{3}\right)(x, t)=\int_{0}^{+\infty} \int_{-\infty}^{+\infty} g_{2}(s) e^{-\gamma_{2} s} \frac{1}{\sqrt{4 \pi d_{3} s}} e^{-\frac{(x-y)^{2}}{4 d_{3} s}} u_{3}(t-s, y) d y d s
\end{gathered}
$$

All the parameters are positive.

The paper is organized as follows: in section 2, we discuss the locally asymptotic stability between the zero balance and the boundary of equilibrium; in section 3, we use the upper-lower solutions and monotone iterative methods to discuss the global stability of the positive equilibrium point.

\section{Equilibrium and Local Asymptotic Stability}

The variables $u_{2}(x, t), u_{3}(x, t)$ of the system (1) have nothing with $u_{1}(x, t)$, so we need to consider the subsystems of the system (1):

In (1), let $u_{2}(x, t)=u(x, t), u_{3}(x, t)=v(x, t), d_{2} \underline{\underline{\Delta}} d_{1}, d_{3} \stackrel{\Delta d_{2}}{=}$, then the system $(1)$ can rewrite following:

$$
\left\{\begin{array}{l}
\frac{\partial u(x, t)}{\partial t}-d_{1} \frac{\partial u^{2}(x, t)}{\partial x^{2}}=\alpha\left(g_{1} * u\right)(x, t)-b_{1} u(x, t)-r_{1} u^{2}(x, t)-c_{1} u(x, t) v(x, t) \\
\frac{\partial v(x, t)}{\partial t}-d_{2} \frac{\partial v^{2}(x, t)}{\partial x^{2}}=\beta\left(g_{2} * v\right)(x, t)-b_{2} v(x, t)-r_{2} v^{2}(x, t)-c_{2} u(x, t) v(x, t)
\end{array}\right.
$$

Lemma 2.1 Assume that $\alpha \int_{0}^{+\infty} g_{1}(s) e^{-\gamma_{1} s} d s>b_{1}, \quad \beta \int_{0}^{+\infty} g_{2}(s) e^{-\gamma_{2} s} d s>b_{2},\left\{\begin{array}{l}k_{1} r_{1}>c_{1} k_{2} \\ k_{2} r_{2}>c_{2} k_{1}\end{array}\right.$ or, $\left\{\begin{array}{l}k_{1} r_{1}<c_{1} k_{2} \\ k_{2} r_{2}<c_{2} k_{1}\end{array}\right.$, then the system(2)has four non-negative equilibrium:

$$
E_{0}=(0,0), E_{1}=\left(\frac{\alpha \int_{0}^{+\infty} g_{1}(s) e^{-\gamma_{1} s} d s-b_{1}}{r_{1}}, 0\right) \Delta\left(k_{1}, 0\right), E_{2}=\left(0, \frac{\beta \int_{0}^{+\infty} g_{2}(s) e^{-\gamma_{2} s} d s-b_{2}}{r_{2}}\right) \underline{\underline{\Delta}}\left(0, k_{2}\right), \quad E=\left(u^{*}, v^{*}\right)
$$

Where,

$$
\begin{aligned}
& u^{*}=\frac{r_{2}\left(\alpha \int_{0}^{+\infty} g_{1}(s) e^{-\gamma_{1} s} d s-b_{1}\right)-c_{1}\left(\beta \int_{0}^{+\infty} g_{2}(s) e^{-\gamma_{2} s} d s-b_{2}\right)}{r_{1} r_{2}-c_{1} c_{2}} \\
& v^{*}=\frac{r_{1}\left(\beta \int_{0}^{+\infty} g_{2}(s) e^{-\gamma_{2} s} d s-b_{2}\right)-c_{2}\left(\alpha \int_{0}^{+\infty} g_{1}(s) e^{-\gamma_{1} s} d s-b_{1}\right)}{r_{1} r_{2}-c_{1} c_{2}}
\end{aligned}
$$

To study the asymptotic stability of the equilibrium by using of constant linear methods similar to [13], We introduce the transformation $U(t)=(u(x, t), v(x, t))-E_{i} \quad(i=1,2)$,

$U_{t}=U(t+\theta)(-\tau \leq \theta \leq 0)$, Then the system (2) Can translate into PFDE of $C \underline{\underline{\Delta} C}\left([-\tau, 0] ; R^{2}\right)$ :

$$
\frac{d}{d t} U(t)=D \Delta U(t)+N(\tau)\left(U_{t}\right)+f_{0}\left(U_{t}, \tau\right)
$$

Where, $D=\operatorname{diag}\left(D_{1}, D_{2}\right), N(\tau): C \rightarrow R^{2}, \quad f_{0}: C \times R^{+} \rightarrow R^{2}$, Then the characteristic equation for the linear part of 
system(3)can become

$$
\left|\begin{array}{cc}
\lambda+k^{2} d_{1}-\alpha \int_{0}^{+\infty} g_{1}(s) e^{-\left(\gamma_{1}+\lambda+d_{1} k^{2}\right) s} d s+b_{1}+2 r_{1} u+c_{1} v & c_{1} u \\
c_{2} v & \lambda+k^{2} d_{2}-\beta \int_{0}^{+\infty} g_{2}(s) e^{-\left(\gamma_{2}+\lambda+d_{2} k^{2}\right) s} d s+b_{2}+2 r_{2} v+c_{2} u
\end{array}\right|=0
$$

Let

$$
\begin{aligned}
& g_{1}\left(\lambda, k^{2}\right)=\lambda+k^{2} d_{1}-\alpha \int_{0}^{+\infty} g_{1}(s) e^{-\left(\gamma_{1}+\lambda+d_{1} k^{2}\right) s} d s+b_{1}+2 r_{1} u+c_{1} v \\
& g_{2}\left(\lambda, k^{2}\right)=\lambda+k^{2} d_{2}-\beta \int_{0}^{+\infty} g_{2}(s) e^{-\left(\gamma_{2}+\lambda+d_{2} k^{2}\right) s} d s+b_{2}+2 r_{2} v+c_{2} u
\end{aligned}
$$

Then the characteristic equation becomes $g_{1}\left(\lambda, k^{2}\right) g_{2}\left(\lambda, k^{2}\right)-c_{1} c_{2} u v=0$,

Theorem 2.1 When $\left\{\begin{array}{l}k_{1} r_{1}>c_{1} k_{2} \\ k_{2} r_{2}<c_{2} k_{1}\end{array}\right.$, the equilibrium $E_{0}=(0,0)$ is unstable.

Proof: for the equilibrium $E_{0}=(0,0)$, the characteristic equation for the system (2) can become $g_{1}\left(\lambda, k^{2}\right) g_{2}\left(\lambda, k^{2}\right)=0$

If $g_{1}\left(\lambda, k^{2}\right)=0$, which yields $\left(\lambda+k^{2} d_{1}+b_{1}-\alpha \int_{0}^{+\infty} g_{1}(s) e^{-\left(\gamma_{1}+\lambda+d_{1} k^{2}\right) s} d s\right)=0$.

So, we get $\left|\lambda+d_{1} k^{2}+b_{1}\right|=\left|\alpha \int_{0}^{+\infty} g_{1}(s) e^{-\left(\gamma_{1}+\lambda+d_{1} k^{2}\right)} d s\right|$,

if $\operatorname{Re} \lambda>0$, then $\left|\lambda+d_{1} k^{2}+b_{1}\right|=\left|\alpha \int_{0}^{+\infty} g_{1}(s) e^{-\left(\gamma_{1}+\lambda+d_{1} k^{2}\right) s} d s\right| \leq \alpha \int_{0}^{+\infty} g_{1}(s) e^{-\gamma_{1} s} d s$ holds.

In the same way, if $g_{2}\left(\lambda, k^{2}\right)=0$, we can have $\operatorname{Re} \lambda>0$.

Thus, $E_{0}=(0,0)$ is unstable.

Theorem 2.2 When $\left\{\begin{array}{l}k_{1} r_{1}>c_{1} k_{2} \\ k_{2} r_{2}<c_{2} k_{1}\end{array}\right.$, the equilibrium $E_{1}$ is local asymptotic stability and $E_{2}$ is unstable.

Proof: for the equilibrium $E_{1}$, the characteristic equation for the system (2) can become $g_{1}\left(\lambda, k^{2}\right) g_{2}\left(\lambda, k^{2}\right)=0$.

(i) If $g_{1}\left(\lambda, k^{2}\right)=0$, which yields, $\lambda+k^{2} d_{1}+b_{1}+2 r_{1} k_{1}=\alpha \int_{0}^{+\infty} g_{1}(s) e^{-\left(\gamma_{1}+\lambda+d_{1} k^{2}\right) s} d s$.

if $\operatorname{Re} \lambda>0$, then

$$
\left|\lambda+k^{2} d_{1}+2 \alpha \int_{0}^{+\infty} g_{1}(s) e^{-\gamma_{1} s} d s\right|=\left|b_{1}+\alpha \int_{0}^{+\infty} g_{1}(s) e^{-\left(\gamma_{1}+\lambda+d_{1} k^{2}\right) s} d s\right| \leq 2 \alpha \int_{0}^{+\infty} g_{1}(s) e^{-\gamma_{1} s} d s
$$

is contradiction with the suppose, so $\operatorname{Re} \lambda \leq 0$;

(ii) if $g_{2}\left(\lambda, k^{2}\right)=0$, then $\lambda+k^{2} d_{2}-\beta \int_{0}^{+\infty} g_{2}(s) e^{-\left(\gamma_{2}+\lambda+d_{2} k^{2}\right) s} d s+b_{2}+c_{2} k_{1}=0$ holds.

if $\operatorname{Re} \lambda>0$, then $\left|\lambda+k^{2} d_{2}+b_{2}+c_{2} k_{1}\right|=\left|\beta \int_{0}^{+\infty} g_{2}(s) e^{-\left(\gamma_{2}+\lambda+d_{2} k^{2}\right) s} d s\right| \leq \beta \int_{0}^{+\infty} g_{2}(s) e^{-\gamma_{2} s} d s$

is contradiction with the suppose, so $\operatorname{Re} \lambda \leq 0$;

Therefore, the equilibrium $E_{1}$ is local asymptotic stability

By similar way, we can prove the balance $E_{2}$ of that the system (2) is not stable.

Theorem 2.3 If $\left\{\begin{array}{l}k_{1} r_{1}>c_{1} k_{2} \\ k_{2} r_{2}>c_{2} k_{1}\end{array}\right.$, the equilibrium $E^{*}$ is local asymptotic stability.

Proof: for the equilibrium $E^{*}$, the characteristic equation for the system (2)can become

$\left(\lambda+d_{1} k^{2}-\alpha \int_{0}^{+\infty} g_{1}(s) e^{-\left(\gamma_{1}+\lambda+d_{1} k^{2}\right) s} d s+b_{1}+2 r_{1} u^{*}+c_{1} v^{*}\right) \cdot\left(\lambda+d_{2} k^{2}-\beta \int_{0}^{+\infty} g_{2}(s) e^{-\left(\gamma_{2}+\lambda+d_{2} k^{2}\right) s} d s+b_{2}+2 r_{2} v^{*}+c_{2} u^{*}\right)-c_{1} c_{2} u^{*} v^{*}=0$.

Since $r_{1} u^{*}+c_{1} v^{*}=\alpha \int_{0}^{+\infty} g_{1}(s) e^{-\gamma_{1} s} d s-b_{1}, \quad r_{2} v^{*}+c_{2} u^{*}=\beta \int_{0}^{+\infty} g_{2}(s) e^{-\gamma_{2} s} d s-b_{2}$, we have 
$\left(\lambda+d_{1} k^{2}+\alpha \int_{0}^{+\infty} g_{1}(s) e^{-\gamma_{1} s} d s-\alpha \int_{0}^{+\infty} g_{1}(s) e^{-\left(\gamma_{1}+\lambda+d_{1} k^{2}\right) s} d s+r_{1} u^{*}\right) \cdot\left(\lambda+d_{2} k^{2}+\beta \int_{0}^{+\infty} g_{2}(s) e^{-\gamma_{2} s} d s-\beta \int_{0}^{+\infty} g_{2}(s) e^{-\left(\gamma_{2}+\lambda+d_{2} k^{2}\right) s} d s+r_{2} v^{*}\right)$
$-c_{1} c_{2} u^{*} v^{*}=0$

let $\lambda=a+b i$

$$
\begin{aligned}
& A_{1}=a+d_{1} k^{2}+r_{1} u^{*}+\alpha \int_{0}^{+\infty} g_{1}(s) e^{-\gamma_{1} s} d s-\alpha \int_{0}^{+\infty} g_{1}(s) e^{-\left(\gamma_{1}+a+d_{1} k^{2}\right)} \cos b s d s \\
& A_{2}=a+d_{2} k^{2}+r_{2} v^{*}+\beta \int_{0}^{+\infty} g_{2}(s) e^{-\gamma_{2} s} d s-\beta \int_{0}^{+\infty} g_{2}(s) e^{-\left(\gamma_{2}+a+d_{2} k^{2}\right)} \cos b s d s \\
& B_{1}=b+\alpha \int_{0}^{+\infty} g_{1}(s) e^{-\left(\gamma_{1}+a+d_{1} k^{2}\right)} \sin b s d s, \quad B_{2}=b+\beta \int_{0}^{+\infty} g_{2}(s) e^{-\left(\gamma_{2}+a+d_{2} k^{2}\right)} \sin b s d s
\end{aligned}
$$

So we can get $\left(A_{1}+B_{1} i\right)\left(A_{2}+B_{2} i\right)=c_{1} c_{2} u^{*} v^{*}$.

which yields $\left\{\begin{array}{l}A_{1} A_{2}-B_{1} B_{2}=c_{1} c_{2} u^{*} v^{*} \\ A_{1} B_{2}+A_{2} B_{1}=0\end{array}\right.$, Further, we get $\left\{\begin{array}{l}A_{1} A_{2}=c_{1} c_{2} u^{*} v^{*}+B_{1} B_{2} \\ A_{1} B_{2}+A_{2} B_{1}=0\end{array}\right.$.

Therefore, $A_{1} A_{2} \leq c_{1} c_{2} u^{*} v^{*}$. if $\operatorname{Re} \lambda \geq 0$, then $A_{1} \geq r_{1} u^{*}, A_{2} \geq r_{2} v^{*}$, so $A_{1} A_{2} \geq r_{1} r_{2} u^{*} v^{*}>0$.

Thereby, $r_{1} r_{2} \leq c_{1} c_{2}$ which is contradiction with the suppose.

Therefore, the equilibrium $E^{*}$ is local asymptotic stability.

The methods are also appropriate for a class of food chain system with stage structure. Such as

$$
\left\{\begin{array}{l}
\dot{x}(t)=T e^{-v t}(x-f)-U_{x^{2}}(t)-a_{1} x(t) y(t) \\
\dot{y}(t)=a_{2} x(t) y(t)-d_{1} y(t)-a_{3} y(t) z(t) \\
\dot{z}(t)=a_{4} y(t) z(t)-d_{2} z(t)
\end{array}\right.
$$

Where, $a_{i}(i=1,2,3,4), d_{i}(i=1,2), T, U, V$ are positive, $x(t)$ represents the population densities of the juvenile and adult at time $\mathrm{t}$ and location $\mathrm{x}, y(t), z(t)$ respectively represent the middle and top predators.

\section{Global Stability}

Using the upper-lower solution method and the monotone iterative method to consider the stability the following equations with the initial-boundary value problem:

$$
\left\{\begin{array}{l}
\frac{\partial u_{1}(x, t)}{\partial t}=d_{1} \frac{\partial^{2} u_{1}(x, t)}{\partial x^{2}}+\alpha\left(\left(g_{1} * u_{1}\right)(x, t)\right)-b_{1} u_{1}(x, t)-r_{1} u_{1}^{2}(x, t)-c_{1} u_{1}(x, t) u_{2}(x, t) \\
\frac{\partial u_{2}(x, t)}{\partial t}=d_{2} \frac{\partial^{2} u_{2}(x, t)}{\partial x^{2}}-b_{2} u_{2}(x, t)-r_{2} u_{2}^{2}(x, t)-c_{2} u_{1}(x, t) u_{2}(x, t)+\beta\left(g_{2} * u_{2}\right)(x, t) \\
u_{i}(t, x)=\varphi_{i}(t, x),(t, x) \in[-\tau, 0] \times[0, \pi] \\
\frac{\partial u_{i}(t, 0)}{\partial x}=\frac{\partial u_{i}(t, \pi)}{\partial x}=0, t>0,(i=1,2)
\end{array}\right.
$$

Definiton 3.1 A pair of smooth function $\tilde{u}=\left(\tilde{u}_{1}, \tilde{u}_{2}\right)$ and $\hat{u}=\left(\hat{u}_{1}, \hat{u}_{2}\right)$ are said to be the coupled upper and lower solutions of problem (4), if $\tilde{u}_{i} \geq \hat{u}_{i}(i=1,2)$ in $[-\tau, 0] \times[0, \pi]$, and the following differential inequalities hold 


$$
\left\{\begin{array}{l}
\frac{\partial \tilde{u}_{1}(x, t)}{\partial t} \geq d_{1} \frac{\partial^{2} \tilde{u}_{1}(x, t)}{\partial x^{2}}+\alpha\left(\left(g_{1} * \tilde{u}_{1}\right)(x, t)\right)-b_{1} \tilde{u}_{1}(x, t)-r_{1} \tilde{u}_{1}^{2}(x, t)-c_{1} \tilde{u}_{1}(x, t) \hat{u}_{2}(x, t) \\
\frac{\partial \tilde{u}_{2}(x, t)}{\partial t} \geq d_{2} \frac{\partial^{2} \tilde{u}_{2}(x, t)}{\partial x^{2}}-b_{2} \tilde{u}_{2}(x, t)-r_{2} \tilde{u}_{2}^{2}(x, t)-c_{2} \hat{u}_{1}(x, t) \tilde{u}_{2}(x, t)+\beta\left(g_{2} * \tilde{u}_{2}\right)(x, t) \\
\frac{\partial \hat{u}_{1}(x, t)}{\partial t} \leq d_{1} \frac{\partial^{2} \hat{u}_{1}(x, t)}{\partial x^{2}}+\alpha\left(\left(g_{1} * \hat{u}_{1}\right)(x, t)\right)-b_{1} \hat{u}_{1}(x, t)-r_{1} \hat{u}_{1}^{2}(x, t)-c_{1} \hat{u}_{1}(x, t) \tilde{u}_{2}(x, t) \\
\frac{\partial \hat{u}_{2}(x, t)}{\partial t} \leq d_{2} \frac{\partial^{2} \hat{u}_{2}(x, t)}{\partial x^{2}}-b_{2} \hat{u}_{2}(x, t)-r_{2} \hat{u}_{2}^{2}(x, t)-c_{2} \tilde{u}_{1}(x, t) \hat{u}_{2}(x, t)+\beta\left(g_{2} * \hat{u}_{2}\right)(x, t)
\end{array}\right.
$$

Lemma 3.1 If there exists a pair of upper and lower $\tilde{u}, \hat{u}$ of problem (4) and $\varphi_{i}(t, x)$ is Hölder continue in $[-\tau, 0] \times[0, \pi]$, then the system (4) has the unique solution $\left(u_{1}(t, x), u_{2}(t, x)\right)$ satisfying $\hat{u}_{i} \leq u_{i} \leq \tilde{u}_{i},(i=1,2)$.

Lemma 3.2 If $\varphi_{i}(t, x)$ is Hölder continue in $[-\tau, 0] \times[0, \pi]$, and $\varphi_{i}(t, x) \geq 0, \varphi_{i}(0, x) \neq 0(i=1,2)$, then the system (4) has the unique solution.

Lemma 3.3 With the assuming of Lemma 3.2, if $u(x, t)$ is the solution of the following problem

$$
\left\{\begin{array}{l}
\frac{\partial u}{\partial t}=D \frac{\partial^{2} u}{\partial x^{2}}+\alpha \int_{0}^{+\infty} \int_{0}^{\pi} g(s) e^{-\gamma s} G(x, y, s) u(t-s, y) d y d s-\beta u^{2}(t, x)+A u(t, x), t>0, x \in[0, \pi] \\
\frac{\partial u}{\partial x}(t, 0)=\frac{\partial u}{\partial x}(t, x), \quad t>0 \\
u(t, x)=\varphi(t, x), \quad(t, x) \in[-\tau, 0] \times[0, \pi]
\end{array}\right.
$$

where, $\alpha, \beta>0, \int_{0}^{+\infty} g(s) d s=1, \quad A \geq 0$, then, $\lim _{t \rightarrow+\infty} u(x, t)=\frac{\alpha \int_{0}^{+\infty} g(s) e^{-\gamma_{s}} d s+A}{\beta}, x \in[0, \pi]$.

Theorem 3.1 If $r_{1} r_{2} \geq c_{1} c_{2}, \varphi_{i}(t, x) \geq 0, \varphi_{i}(0, x) \neq 0(i=1,2)$, and let $\left(u_{1}(t, x), u_{2}(t, x)\right)$ be the solution of the system (4), then $\lim _{t \rightarrow+\infty}\left(u_{1}(x, t), u_{2}(x, t)\right)=\left(u_{1}^{*}, u_{2}^{*}\right)$, uniformly for $x \in[0, \pi]$.

Proof: let $K_{1}=\lim _{t \rightarrow+\infty} \sup \max u_{x \in[0, \pi]}(x, t), \quad K_{2}=\lim _{t \rightarrow+\infty} \sup \max u_{x \in[0, \pi]}(x, t)$

Let $\bar{u}_{1}^{(1)}(t, x), \bar{u}_{2}^{(1)}(t, x)$ be the solutions of

$$
\left\{\begin{array}{l}
\frac{\partial \bar{u}_{1}^{(1)}(x, t)}{\partial t}=d_{1} \frac{\partial^{2} \bar{u}_{1}^{(1)}(x, t)}{\partial x^{2}}+\alpha\left(\left(g_{1} * \bar{u}_{1}^{(1)}\right)(x, t)\right)-b_{1} \bar{u}_{1}^{(1)}(x, t)-r_{1}\left[\bar{u}_{1}^{(1)}\right]^{2}(x, t) \\
\frac{\partial \bar{u}_{2}^{(1)}(x, t)}{\partial t}=d_{2} \frac{\partial^{2} \bar{u}_{2}^{(1)}(x, t)}{\partial x^{2}}-b_{2} \bar{u}_{2}^{(1)}(x, t)-r_{2}\left[\bar{u}_{2}^{(1)}\right]^{2}(x, t)+\beta\left(g_{2} * \bar{u}_{2}^{(1)}\right)(x, t) \\
\bar{u}_{1}^{(1)}(t, x)=K_{1}, \bar{u}_{2}^{(1)}(t, x)=K_{2}
\end{array}\right.
$$

Clearly $\left(\bar{u}_{1}^{(1)}(t, x), \bar{u}_{2}^{(1)}(t, x)\right)$ and $(0,0)$ are the upper and lower solutions of problem (4), and by Lemma 3.1, we get $0 \leq u_{i} \leq \bar{u}_{i}^{(1)},(i=1,2)$

On the other hand, by Lemma 3.3, we have

$$
\lim _{t \rightarrow+\infty} \bar{u}_{1}^{(1)}(t, x)=\frac{\alpha \int_{0}^{+\infty} g_{1}(s) e^{-\gamma_{1} s} d s-b_{1}}{r_{1}} \Delta \beta_{1}^{(0)}, \lim _{t \rightarrow+\infty} \bar{u}^{(1)}(t, x)=\frac{\beta \int_{0}^{+\infty} g_{2}(s) e^{-\gamma_{2} s} d s-b_{2}}{r_{2}} \Delta \beta_{2}^{(0)}
$$

And thus for any sufficiently small $\varepsilon>0$, there exists $t_{1}>0$, such that $t>t_{1}$,

$$
\max _{x \in[0, \pi]} u_{1}(x, t)<\beta_{1}^{(0)}+\mathcal{E}, \max _{x \in[0, \pi]} u_{2}(x, t)<\beta_{2}^{(0)}+\mathcal{E}
$$


Let $\underline{u}_{1}^{(1)}(t, x), \underline{u}_{2}^{(1)}(t, x)$ be the solutions of

$$
\left\{\begin{array}{l}
\frac{\partial \underline{u}_{1}^{(1)}}{\partial t}=d_{1} \frac{\partial^{2}{\underline{u_{1}}}^{(1)}}{\partial x^{2}}+\alpha\left(\left(g_{1} * \underline{u}_{1}^{(1)}\right)\right)-b_{1} \underline{u}_{1}^{(1)}-r_{1}\left[\underline{u_{1}}{ }^{(1)}\right]^{2}-c_{1} \underline{u}_{1}^{(1)} \bar{u}_{2}^{(1)} \\
\frac{\partial \underline{u}_{2}^{(1)}}{\partial t}=d_{2} \frac{\partial^{2}{\underline{u_{2}}}^{(1)}}{\partial x^{2}}-b_{2} \underline{u}^{(1)}-r_{2}\left[\underline{u}_{2}^{(1)}\right]^{2}+\beta\left(g_{2} * \underline{u}_{2}{ }^{(1)}\right)-c_{2} \bar{u}_{1}{ }^{(1)} \underline{u}_{2}{ }^{(1)} \\
\underline{u}^{(1)}(t, x)=\frac{1}{2} u_{1}(t, x), \underline{u}_{2}^{(1)}(t, x)=\frac{1}{2} u_{2}(t, x)
\end{array}\right.
$$

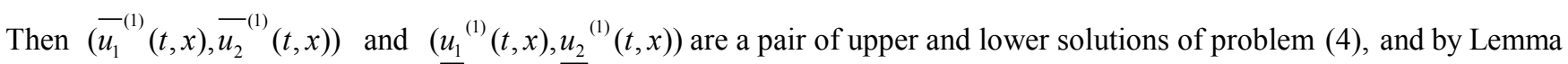
3.1 , we get $\underline{u}_{i}^{(1)} \leq u_{i} \leq \bar{u}_{i}^{(1)},(i=1,2)$

By (7) and (8), we have

$$
\left\{\begin{array}{l}
\frac{\partial{\underline{u_{1}}}^{(1)}}{\partial t} \geq d_{1} \frac{\partial^{2}{\underline{u_{1}}}^{(1)}}{\partial x^{2}}+\alpha\left(\left(g_{1} * \underline{u}_{1}{ }^{(1)}\right)\right)-b_{1} \underline{u}_{1}^{(1)}-r_{1}\left[\underline{u}_{1}^{(1)}\right]^{2}-c_{1} \underline{u}_{1}^{(1)}\left(\beta_{2}^{(0)}+\varepsilon\right) \\
\frac{\partial \underline{u}_{2}{ }^{(1)}}{\partial t}=d_{2} \frac{\partial^{2} \underline{u}_{2}^{(1)}}{\partial x^{2}}-b_{2} \underline{u}_{2}{ }^{(1)}-r_{2}\left[\underline{u}_{2}{ }^{(1)}\right]^{2}+\beta\left(g_{2} \underline{u}_{2}{ }^{(1)}\right)-c_{2} \underline{u}_{2}{ }^{(1)}\left(\beta_{1}^{(0)}+\varepsilon\right)
\end{array}\right.
$$

By the comparison principle, we get ${\underline{u_{1}}}^{(1)} \geq v_{1}{ }^{(1)},{\underline{u_{2}}}^{(1)} \geq v_{2}{ }^{(1)}$, where $v_{1}^{(1)}$ and $v_{2}{ }^{(1)}$ are the upper and lower solutions of problem

$$
\left\{\begin{array}{l}
\frac{\partial v_{1}^{(1)}}{\partial t}=d_{1} \frac{\partial^{2} v_{1}^{(1)}}{\partial x^{2}}+\alpha\left(\left(g_{1} * v_{1}^{(1)}\right)\right)-b_{1} v_{1}^{(1)}-r_{1}\left[\underline{v_{1}{ }^{(1)}}\right]^{2}-c_{1} v_{1}^{(1)}\left(\beta_{2}^{(0)}+\varepsilon\right) \\
\frac{\partial v_{2}^{(1)}}{\partial t}=d_{2} \frac{\partial^{2} \underline{v}^{(1)}}{\partial x^{2}}-b_{2} \underline{v}_{2}^{(1)}-r_{2}\left[\underline{v}_{2}^{(1)}\right]^{2}+\beta\left(g_{2} * \underline{v}_{2}{ }^{(1)}\right)-c_{2} v_{2}{ }^{(1)}\left(\beta_{1}^{(0)}+\varepsilon\right) \\
v_{1}^{(1)}=\frac{1}{2} u_{1}(t, x), v_{2}^{(1)}=\frac{1}{2} u_{2}(t, x)
\end{array}\right.
$$

by Lemma 3.3, we have

$$
\lim _{t \rightarrow+\infty} v_{1}^{(1)}(t, x)=\frac{\alpha \int_{0}^{+\infty} g_{1}(s) e^{-\gamma_{1} s} d s-b_{1}-c_{1}\left(\beta_{2}^{(0)}+\varepsilon\right)}{r_{1}}, \quad \lim _{t \rightarrow+\infty} v_{2}^{(1)}(t, x)=\frac{\beta \int_{0}^{+\infty} g_{2}(s) e^{-\gamma_{2} s} d s-b_{2}-c_{2}\left(\beta_{1}^{(0)}+\varepsilon\right)}{r_{2}}
$$

Therefore, we can conclude that

$$
\begin{aligned}
& 0<\alpha_{1}^{(0)} \leq \lim _{t \rightarrow+\infty} \inf \min _{x \in[0, \pi]} u_{1}(t, x) \leq \lim _{t \rightarrow+\infty} \sup \max _{x \in[0, \pi]} u_{1}(t, x) \leq \beta_{1}^{(0)} \\
& 0<\alpha_{2}^{(0)} \leq \lim _{t \rightarrow+\infty} \inf \min _{x \in[0, \pi]} u_{2}(t, x) \leq \lim _{t \rightarrow+\infty} \sup _{x \in[0, \pi]} \max _{2} u_{2}(t, x) \leq \beta_{2}^{(0)}
\end{aligned}
$$

Where, $\alpha_{1}^{(0)}=\frac{\alpha \int_{0}^{+\infty} g_{1}(s) e^{-\gamma_{1} s} d s-b_{1}-c_{1} \beta_{2}^{(0)}}{r_{1}}, \alpha_{2}^{(0)}=\frac{\beta \int_{0}^{+\infty} g_{2}(s) e^{-\gamma_{2} s} d s-b_{2}-c_{2} \beta_{1}^{(0)}}{r_{2}}$

Furthermore, for any sufficiently small $\varepsilon>0$, there exists $t>t_{2}$, such that

$$
\min _{x \in[0, \pi]} \underline{u}_{1}^{(1)}(x, t)>\alpha_{1}^{(0)}-\varepsilon, \min _{x \in[0, \pi]} \underline{u}_{2}^{(1)}(x, t)>\alpha_{2}^{(0)}-\varepsilon, t>t_{2}
$$

Let $\bar{u}_{1}^{(2)}(t, x), \bar{u}_{2}^{(2)}(t, x)$ be the solutions of 


$$
\left\{\begin{array}{l}
\frac{\partial \bar{u}_{1}^{(2)}}{\partial t}=d_{1} \frac{\partial^{2} \bar{u}_{1}^{(2)}}{\partial x^{2}}+\alpha\left(\left(g_{1} * \bar{u}_{1}^{(2)}\right)\right)-b_{1} \bar{u}_{1}^{(2)}-r_{1}\left[\bar{u}_{1}^{(2)}\right]^{2}-c_{1} \bar{u}_{1}^{(2)} \underline{u}_{2}^{(1)} \\
\frac{\partial \bar{u}^{(2)}}{\partial t}=d_{2} \frac{\partial^{2} \bar{u}^{(2)}}{\partial x^{2}}-b_{2} \bar{u}_{2}^{(2)}-r_{2}\left[\bar{u}^{(2)}\right]^{2}+\beta\left(g_{2} * \bar{u}_{2}^{(2)}\right)-c_{2} \underline{u}_{1}^{(1)} \bar{u}_{2}^{(2)} \\
\bar{u}_{1}^{(2)}(t, x)=K_{1}, \bar{u}^{(2)}(t, x)=K_{2}
\end{array}\right.
$$

\left. By definition 3.1, ${\overline{\left(u_{1}\right.}}^{(2)}(t, x), \bar{u}_{2}{ }^{(2)}(t, x)\right)$ and ${\left(u_{1}\right.}^{(1)}(t, x), u_{2}{ }^{(1)}(t, x))$ are a pair of upper and lower solutions of problem (4), and by Lemma 3.1, we get $\underline{u}_{i}^{(1)} \leq u_{i} \leq \bar{u}_{i}^{(2)},(i=1,2)$

By (12) and (13), we have

$$
\left\{\begin{array}{l}
\frac{\partial \bar{u}_{1}^{(2)}}{\partial t} \leq d_{1} \frac{\partial^{2} \bar{u}_{1}^{(2)}}{\partial x^{2}}+\alpha\left(\left(g_{1} * \bar{u}_{1}^{(2)}\right)\right)-b_{1} \bar{u}_{1}^{(2)}-r_{1}\left[\bar{u}_{1}^{(2)}\right]^{2}-c_{1} \bar{u}_{1}^{(2)}\left(\alpha_{2}^{(0)}-\varepsilon\right) \\
\frac{\partial \bar{u}^{(2)}}{\partial t} \leq d_{2} \frac{\partial^{2} \bar{u}_{2}^{(2)}}{\partial x^{2}}-b_{2} \bar{u}_{2}^{(2)}-r_{2}\left[\bar{u}^{(2)}\right]^{2}+\beta\left(g_{2} * \bar{u}_{2}^{(2)}\right)-c_{2} \bar{u}_{2}^{(2)}\left(\alpha_{1}^{(0)}-\varepsilon\right)
\end{array}\right.
$$

By the comparison principle, we get $\bar{u}_{1}^{(2)} \leq w_{1}^{(1)}, \bar{u}_{2}^{(2)} \leq w_{2}^{(1)}$, where $w_{1}^{(1)}, w_{2}^{(1)}$ are the upper and lower solutions of problem

$$
\left\{\begin{array}{l}
\frac{\partial w_{1}^{(1)}}{\partial t}=d_{1} \frac{\partial^{2} w_{1}^{(1)}}{\partial x^{2}}+\alpha\left(\left(g_{1} * \partial w_{1}^{(1)}\right)\right)-b_{1} w_{1}^{(1)}-r_{1}\left[w_{1}^{(1)}\right]^{2}-c_{1} w_{1}^{(1)}\left(\alpha_{2}^{(0)}-\varepsilon\right) \\
\frac{\partial w_{2}^{(1)}}{\partial t}=d_{2} \frac{\partial^{2} w_{2}^{(1)}}{\partial x^{2}}-b_{2} w_{2}^{(1)}-r_{2}\left[w_{2}{ }^{(1)}\right]^{2}+\beta\left(g_{2} * w_{2}^{(1)}\right)-c_{2} w_{2}^{(1)}\left(\alpha_{1}^{(0)}-\varepsilon\right) \\
w_{1}^{(1)}=K_{1}, w_{2}^{(1)}=K_{2}
\end{array}\right.
$$

by Lemma 3.3, we have

$$
\lim _{t \rightarrow+\infty} w_{1}^{(1)}(t, x)=\frac{\alpha \int_{0}^{+\infty} g_{1}(s) e^{-\gamma_{1} s} d s-b_{1}-c_{1}\left(\alpha_{2}^{(0)}-\varepsilon\right)}{r_{1}}, \lim _{t \rightarrow+\infty} w_{2}^{(1)}(t, x)=\frac{\beta \int_{0}^{+\infty} g_{2}(s) e^{-\gamma_{2} s} d s-b_{2}-c_{2}\left(\alpha_{1}^{(0)}-\varepsilon\right)}{r_{2}}
$$

Therefore we can conclude that

$$
\begin{aligned}
& 0<\alpha_{1}^{(0)} \leq \lim _{t \rightarrow+\infty} \inf \min _{x \in[0, \pi]} u_{1}(t, x) \leq \lim _{t \rightarrow+\infty} \sup _{\max _{x \in[0, \pi]} u_{1}(t, x) \leq \beta_{1}^{(1)}} \\
& 0<\alpha_{2}^{(0)} \leq \lim _{t \rightarrow+\infty} \inf \min _{x \in[0, \pi]} u_{2}(t, x) \leq \lim _{t \rightarrow+\infty} \sup \max _{x \in[0, \pi]} u_{2}(t, x) \leq \beta_{2}^{(1)}
\end{aligned}
$$

where, $\beta_{1}^{(1)}=\frac{\alpha \int_{0}^{+\infty} g_{1}(s) e^{-\gamma_{1} s} d s-b_{1}-c_{1} \alpha_{2}^{(0)}}{r_{1}}, \beta_{2}^{(1)}=\frac{\beta \int_{0}^{+\infty} g_{2}(s) e^{-\gamma_{2} s} d s-b_{2}-c_{2} \alpha_{1}^{(0)}}{r_{2}}$

It is obvious that $0<\alpha_{1}^{(0)} \leq \beta_{1}^{(1)} \leq \beta_{1}^{(0)}, \quad 0<\alpha_{2}^{(0)} \leq \beta_{2}^{(1)} \leq \beta_{2}^{(0)}$.

Continue this process, we can get the following sequences

$$
\begin{aligned}
\alpha_{1}^{(k)} & =\frac{\alpha \int_{0}^{+\infty} g_{1}(s) e^{-\gamma_{1} s} d s-b_{1}-c_{1} \beta_{2}^{(k)}}{r_{1}}, \alpha_{2}{ }^{(k)}=\frac{\beta \int_{0}^{+\infty} g_{2}(s) e^{-\gamma_{2} s} d s-b_{2}-c_{2} \beta_{1}^{(k)}}{r_{2}} \\
\beta_{1}^{(k+1)} & =\frac{\alpha \int_{0}^{+\infty} g_{1}(s) e^{-\gamma_{1} s} d s-b_{1}-c_{1} \alpha_{2}^{(k)}}{r_{1}}, \beta_{2}^{(k+1)}=\frac{\beta \int_{0}^{+\infty} g_{2}(s) e^{-\gamma_{2} s} d s-b_{2}-c_{2} \alpha_{1}^{(k)}}{r_{2}}
\end{aligned}
$$




$$
\beta_{1}^{(0)}=\frac{\alpha \int_{0}^{+\infty} g_{1}(s) e^{-\gamma_{1} s} d s-b_{1}}{r_{1}}, \beta_{2}^{(0)}=\frac{\beta \int_{0}^{+\infty} g_{2}(s) e^{-\gamma_{2} s} d s-b_{2}}{r_{2}}
$$

And satisfying

$$
\begin{aligned}
& 0<\alpha_{1}^{(k)} \leq \lim _{t \rightarrow+\infty} \inf \min _{x \in[0, \pi]} u_{1}(t, x) \leq \lim _{t \rightarrow+\infty} \sup _{x \in[0, \pi]} \max _{1} u_{1}(t, x) \leq \beta_{1}^{(k)} \\
& 0<\alpha_{2}^{(k)} \leq \lim _{t \rightarrow+\infty} \inf \min _{x \in[0, \pi]} u_{2}(t, x) \leq \lim _{t \rightarrow+\infty} \sup _{x \in[0, \pi]} \max _{2}(t, x) \leq \beta_{2}^{(k)} \\
& {\left[\alpha_{1}^{(k+1)}, \beta_{1}^{(k+1)}\right] \subseteq\left[\alpha_{1}^{(k)}, \beta_{1}^{(k)}\right], \quad\left[\alpha_{2}^{(k+1)}, \beta_{2}^{(k+1)}\right] \subseteq\left[\alpha_{2}^{(k)}, \beta_{2}^{(k)}\right]}
\end{aligned}
$$

We need to testify the following $\alpha_{1}=\beta_{1}=u_{1}^{*}, \quad \alpha_{2}=\beta_{2}=u_{2}^{*}$

Let $k \rightarrow+\infty$ in (17), we derive that

$$
\left\{\begin{array}{l}
\alpha_{1} r_{1}+c_{1} \beta_{2}=\alpha \int_{0}^{+\infty} g_{1}(s) e^{-\gamma_{1} s} d s-b_{1} \\
\alpha_{2} r_{2}+c_{2} \beta_{1}=\beta \int_{0}^{+\infty} g_{2}(s) e^{-\gamma_{2} s} d s-b_{2} \\
r_{1} \beta_{1}+c_{1} \alpha_{2}=\alpha \int_{0}^{+\infty} g_{1}(s) e^{-\gamma_{1} s} d s-b_{1} \\
r_{2} \beta_{2}+c_{2} \alpha_{1}=\beta \int_{0}^{+\infty} g_{2}(s) e^{-\gamma_{2} s} d s-b_{2}
\end{array}\right.
$$

Which yields $\left\{\begin{array}{l}\left(\alpha_{1}-\beta_{1}\right) r_{1}-c_{1}\left(\alpha_{2}-\beta_{2}\right)=0 \\ \left(\alpha_{2}-\beta_{2}\right) r_{2}-c_{2}\left(\alpha_{1}-\beta_{1}\right)=0\end{array}\right.$

since $\left|\begin{array}{cc}r_{1} & -c_{1} \\ -c_{2} & r_{2}\end{array}\right|=r_{1} r_{2}-c_{1} c_{2}>0$, system (4) has only zero solution with respect to $\alpha_{1}=\beta_{1}, \alpha_{2}=\beta_{2}$. Therefore, from(18), we can get $\alpha_{1}=\beta_{1}=u_{1}^{*}, \alpha_{2}=\beta_{2}=u_{2}^{*}$.

This completes the proof.

The methods are also appropriate for a class of cooperation model and Epidemic model with stage structure, and so on. for example:

$$
\left\{\begin{array}{l}
\frac{\partial u_{1}(x, t)}{\partial t}-d_{1} \frac{\partial^{2} u_{1}(x, t)}{\partial x^{2}}=\alpha\left(\left(g_{1} * u_{1}\right)(x, t)\right)-r_{1} u_{1}^{2}(x, t)+c_{1} u_{1}(x, t) u_{2}(x, t) \\
\frac{\partial u_{2}(x, t)}{\partial t}-d_{2} \frac{\partial^{2} u_{2}(x, t)}{\partial x^{2}}=\beta\left(\left(g_{2} * u_{2}\right)(x, t)\right)-r_{2} u_{2}^{2}(x, t)+c_{2} u_{1}(x, t) u_{2}(x, t)
\end{array}\right.
$$

Where, the parameters have the same the meanings with consistent.

\section{Conclusion}

Using of constant linear methods, we considered the local asymptotic stability; Employing the upper-lower solutions and monotone iterative methods, we considered the global stability of the positive equilibrium point about the competition model with diffusion terms and stage structure. The conclusions are also appropriate for the corresponding parabolic-ordinary differential system ( $d_{i}=0$ for some or all $i$ ). Besides, The conclusions are also appropriate for the predator-prey model and epidemic model and so on. So, The traditional results are improved and this result adds to the previous results and applies to broader frameworks. But, with the increase of the invasive species, we can study the multi-group reaction diffusion model in the next few years.

\section{Acknowledgments}

The paper was funded by the field incubation project 2015 L02.

\section{References}

[1] Chen Lansun, The phase structure of the population dynamics model $[\mathrm{J}]$. Journal of north China university (natural science edition), in June 2000, 1 (3): 185-191. 
[2] Wang Lie, Chen Siyang, Infected with time delay and stage structure vaccination of youth the stability of the single population model [J]. Journal of Shan xi normal university (natural science edition), in January, 2013, 41 (1): 15-19.

[3] Yan Weilin, Two species competition model with stage structure of the optimal harvesting [J]. Journal of Shanxi university (natural science edition), 2013, 29 (5) in October: $10-12$.

[4] Shi Chunling, Global attractivity in a Lotka-Volterra competition system with feedback controls [J]. Journal of Biomathematics, 2015, 30 (4): 714-720.

[5] Li Liangchen, Xu Rui, Global stability and bifurcations of a delayed predator-prey model with Holling type II functional response [J]. Journal of natural science of Heilongiiang university, 2016, 33 (3) in June: 328-337.

[6] Gao Shujing, Global stability of three stage structured single species growth model [J]. Journal of Xinjiang university: Science Edition, 2001, 18 (2): 154-158.

[7] Liang Zhiqing, Zhaoqiang, The optimal harvesting strategy about a class of single population model with three phase structure $[\mathrm{J}]$. Journal of Yulin normal university (natural science edition), 2005, 26 (3): 1-3.
[8] Wu Chufen, Global asymptotic stability of a weakly-coupled reaction diffusion system in the three-species model [J]. Journal of South China normal university (natural science edition), 2015, 47 (3): 142-147.

[9] Mou En, Zhao Chaofeng, Chen Xiaodong, Zhang Qimin, Stability for single population growth model with three-stage structure and time delay [J]. Journal of Chongqing normal university (natural science), 2016, 33 (4): 85-89.

[10] Gao Shujing, Chen Lansun, Permanence and global stability for single species model with three life stages and time delay [J]. Acta Mathematica Science, 2006, 26A (4): 527-533.

[11] Li Yuxia, Global asymptotic stability in three-species non-autonomous system with time-delay [J]. Journal of Taiyuan normal university (natural science), 2016, 15 (2): 26-28.

[12] Song Lingyu, Liu Fumin, Two predator-prey model with stage structure with diffusion is continuous existence of approximate wave-front solutions [J]. Journal of engineering mathematics, 28 October 2011 (5): 671-680.

[13] Faria T. Stability and bifurcation for a delayed predator-prey model and the effect of diffusion [J]. Journal of Mathematical Analysis and Applications, 2001, 254: 433-463.5. 